\title{
Thérapies antisens dirigées contre le récepteur de l'IGF-I
}

Plusieurs études ont révélé que le récepteur de type I des IGF (insulin-like growth factors) contribue au processus de tumorigenèse. L'inhibition de l'expression ou de la fonction de ce récepteur au sein de cellules tumorales a été obtenue avec succès par différen tes approches, notamment par l'utilisation d'ARN ou d'oligonucléotides antisens. Ceux-ci sont en effet capables, dans des modèles expérimentaux, de bloquer l'expression du récepteur au sein de cellules de glioblastomes et d'inhiber leur tumorigen èse in vivo en induisant I'apoptose cellulaire et/ ou une réponse immunitaire. Ces approches antisen s de contrôle de l'expression du récepteur des IGF sont actu ellement à l'étu de chez I'homme dans des essais cliniques préliminaires.
$\mathbf{L}$ es anomalies de la croissance cellulaire par modification de l'expression de facteurs de croissance ou de leurs récepteurs sont des facteurs importants de la progression tumorale [1]. Parmi les facteurs de croissance pouvant être impliqués dans la tumorigenèse se trouvent les protéines de la famille des IGF (insulin-like growth factors) [2]. Ces peptides reconnaissent une protéine transmembranaire, le récepteur de type I des IGF (IGF-IR) doté d'une activité tyrosine kinase activable par la fixation des ligands [3]. Le ligand fixé sur son récepteur déclenche alors I'activation de plusieurs cascades de signalisation intracellulaire qui impliquent, entre autres, la voie des MAP kinases (mitogen activated protein) et celle des PI3 kinases (phosphatidylinositol-3) (figure 1) [2]. Grâce à l'activation de ces voies de signalisations intracellulaires, I'IGF-IR contrôle la croissance, la différentiation cellulaire et la maintenance du phénotype transformé des cellules malignes [3]. Le récepteur agit également en protégeant la cellule de l'apoptose et peut donc être considéré comme une protéine de «survie» permettant le développement de la cellule tumorale [3]. L'IGF-IR et ses ligands sont souvent surexprimés dans certaines lignées cancéreuses animales et dans $\mathrm{m} / \mathrm{s} n^{\circ} 12$, vol. 17 , décembre 2001 quelques tumeurs humaines [2, 4]. Par exemple, chez la femme, I'expression de I'IGF-IR est 10 à 14 fois plus forte dans les cancers du sein que dans les tissus normaux [5]. II apparaît donc intéressant de bloquer la fonction ou l'expression de l'IGF-I ou de son récepteur IGF-IR afin d'inhiber la croissance tumorale in vivo. A titre indicatif, les différentes stratégies expérimentales utilisées pour inhiber la voie de signalisation de IGF-IR sont

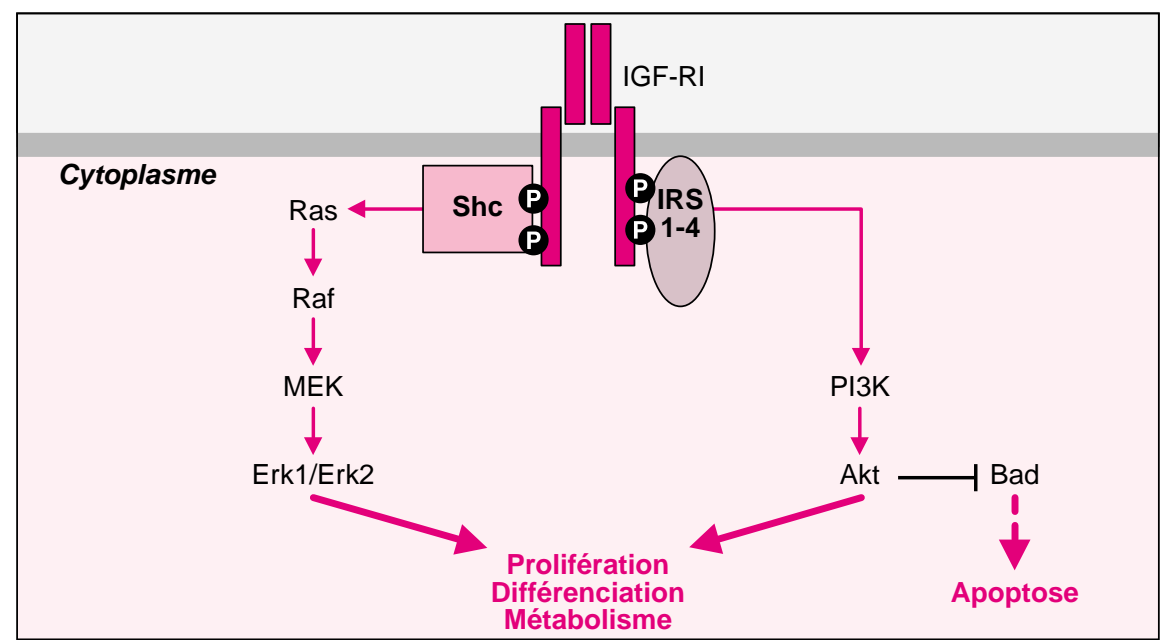

Figure 1. Voies de signalisation du récepteur de I'IGF-I. La liaison de I'IGF-I sur son récepteur, IGF-IR, provoque la phosphorylation des tyrosines du récepteur qui peut alors activer par phosphorylation plusieurs substrats comme l'adaptateur Shc et les protéines IRS (insulin receptor substrate). Ces protéines activent alors d'autres protéines en aval comme les kinases MEK (mitogen extracellular regulated kinase) puis ERK (extracellular regulated kinase) et la voie de signalisation de la PI3-K. Toutes ces cascades peuvent conduire à la prolifération, à la différenciation et à l'augmentation du métabolisme. La protéine kinase Akt activée par I'IGF-IR phosphoryle et inactive la protéine pro-apoptotique Bad, ce qui inhibe I'apoptose cellulaire (pour revue [2, 3]). 


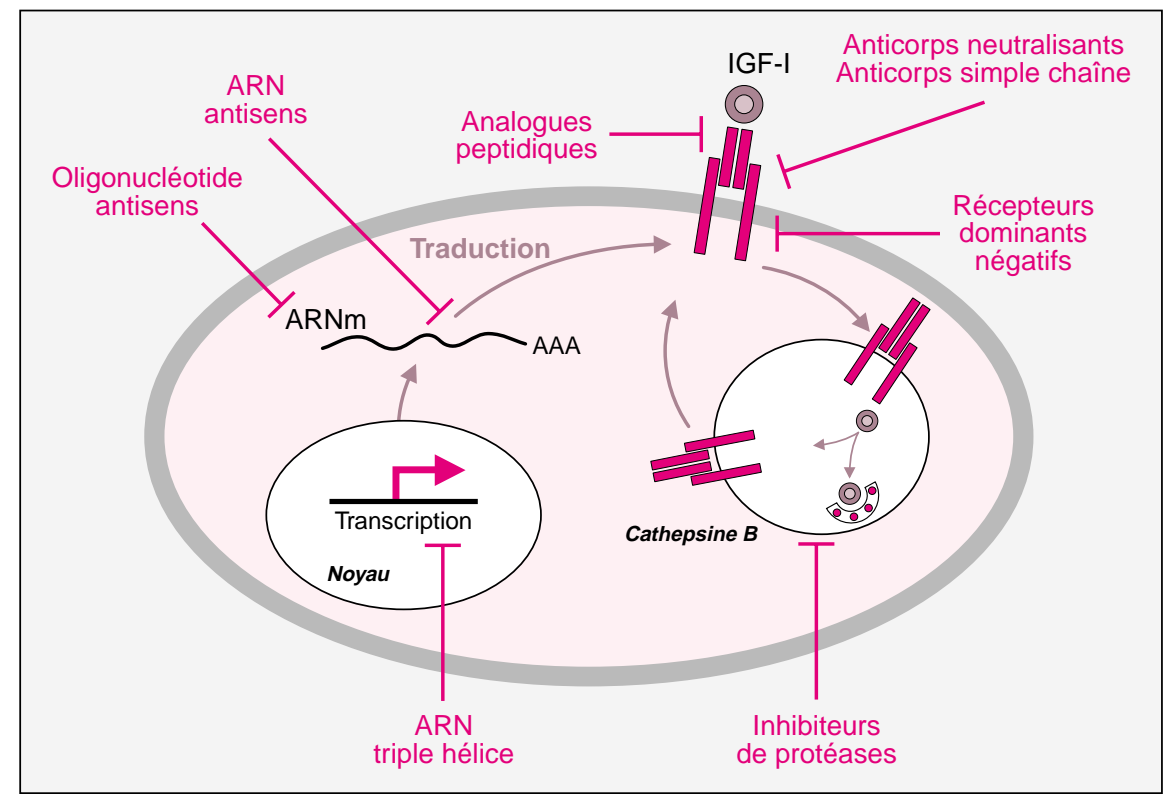

Figure 2. Différentes approches utilisées pour inhiber l'expression du récepteur de I'IGF-I. Le récepteur de I'IGF-I (IGF-IR) est un hétérotétramère $\alpha 2 \beta 2$ composé de sous unités liées par des ponts disulfures. Des anticorps neutralisants et des anticorps simple chaine dirigés contre la partie extracellulaire $\alpha$ d'IGF-IR ont été utilisés pour inhiber la croissance tumorale [22]. Des récepteurs dominants négatifs correspondant à des protéines tronquées soit sur la sous unité intracellulaire $(\beta \mathrm{m})$ soit sur la sous-unité extracellulaire $(\alpha \mathrm{m})$ bloquent également la fonction d'IGF-IR $[23,24]$. Des analogues peptidiques de I'IGF-I inhibent la signalisation induite par IGF-IR en entrant en compétition avec le ligand IGF-I [25]. La fonction de I'IGF-IR peut aussi être bloquée par I'utilisation d'inhibiteurs spécifiques de protéases impliquées dans le recyclage du récepteur [26]. L'inhibiteur de protéase bloque la dégradation du ligand IGF-I normalement effectuée par la cathepsine B. Trois autres stratégies utilisant des acides nucléiques ont été utilisées pour inhiber l'expression d'IGF-IR. Les ARN antisens qui, en se fixant sur l'ARN messager du récepteur, bloquent sa traduction en protéine $[9,10,12-14,17,19,20]$. Les oligonucléotides antisens ont le même effet, ceci grâce à l'induction d'une ribonucléase spécifique, la RNase $H[6,10,15,27]$. Des ARN peuvent aussi être dirigés contre I'ADN de I'IGF-I ou de I'IGF-IR via la formation de triple hélice afin d'inhiber leur transcription [11, 28].

présentées sur la figure 2. Les approches utilisant les ARN ou les oligonucléotides antisens dirigés contre les ARN messagers de I'IGF-IR ont conduit très récemment à des essais cliniques préliminaires [6].

\section{Les ARN et oligonucléotides antisens}

Les ARN et les oligonucléotides antisens inhibent la traduction de l'ARN messager sur lequel ils sont dirigés. Brièvement, I'ARN antisens complémentaire de I'ARN m, en formant un ARN double brin, bloque physique- dis que les oligonucléotides antisens induisent, la plupart du temps, une ribonucléase spécifique, la $\mathrm{RN}$ ase $\mathrm{H}$, qui reconnaît l'hybride ARN m/ oligonucléotide et dégrade le brin ribonucléique (pour revue, $[7,8]$ ). L'ARN antisens est généralement synthétisé à partir de constructions plasmidiques qui peuvent être introduites dans les cellules tumorales soit in vitro par une transfection cellulaire avec des lipides cationiques [9-12], soit in vivo à l'aide d'un vecteur adénoviral [13] ou bien par injection directe d'ADN nu plasmidique au site de la tumeur [14].
Les oligonucléotides antisens qui représentent une bonne alternative aux ARN antisens peuvent être obtenus par synthèse chimique et pénètrent spontanément dans les cellules. Afin d'augmenter leurs effets biologiques, il apparaît parfois nécessaire d'une part de les rendre résistants vis-à-vis des nucléases biologiques en les modifiant chimiquement, et d'autre part d'ajouter des agents de transfection pour augmenter leur concentration intracellulaire. II faut souvent adapter le couple oligonucléotide modifié et agent de tran sfection au type cellulaire que l'on désire transfecter in vitro [8]. Toutefois, les oligonucléotides antisens sont parfois utilisés sans addition de vecteurs de transport pour des expériences chez I'animal $[10,15]$. Une revue récente pourra être consultée pour obtenir des détails sur les méthodes utilisées pour délivrer les oligonucléotides au sein des cellules et/ ou des tissus [16].

Divers oligonucléotides antisens ont été dirigés contre l'ARNm de I'IGFIR et ont été capables d'inhiber l'expression et la fonction du récepteur aussi bien in vitro qu'in vivo. Par exemple, il a été montré que des injections intradermiques répétées d'oligonucléotides antisens anti-IGFIR provoquaient une régression significative de l'hyperplasie épidermique observée chez des souris nude greffées avec de la peau humaine atteinte de psoriasis [15]. L'IGF-IR est en effet fortement exprimé au sein des lésions de psoriasis et représentait donc une cible potentiellement intéressante dans le cadre de cette pathologie.

\section{Récepteur de type I des IG F et glioblastomes}

L'utilisation d'approches antisens a permis de démontrer que le récepteur de I'IGF-I jouait un rôle important dans la tumorigenèse in vivo de glioblastomes de rat (pour revue voir [17]). Le modèle utilisé est le développement de tumeurs après injection de cellules de glioblastome (C6) à des rats immuno-compétents $\mathrm{BD}-\mathrm{IX}$ (figure 3). Les cellules de glioblastome de rat transfectées par un plasmide codant pour l'ARN antisens de I'IGF- 
IR (cellules C6T) n'expriment plus le récepteur et $n$ 'induisent plus la formation de tumeurs in vivo. Elles provoquent de plus une réponse antitumorale in vivo, protégeant le rat de I'apparition de tumeurs qui seraient induites après une seconde injection de cellules non transfectées [10,17]. Enfin, des tumeurs déjà établies régressent après injection de ces celIules C6T (figure 3). Le fait d'inhiber I'IGF-IR dans une lignée tumorale a donc permis de disposer d'une sorte de vaccin cellulaire anti-tumoral empêchant tout développement ultérieur de tumeurs de même origine in vivo. II est à noter que les modèles animaux utilisés dans ces expériences d'ARN antisens anti IGF-IR et anti IGF-I (voir paragraphe suivant) ne sont pas syngéniques avec les glioblastomes $\mathrm{C} 6$ et que la greffe peut être considérée comme allogénique [18]. Des effets biologiques similaires ont été observés lorsque les cellules C6 ont été préalablement traitées in vitro avec un oligonucléotide phosphorothioate antisens dirigé contre le codon d'initiation de la traduction du récepteur de I'IGF-I et réimplantées chez le rat soit directement, soit à l'aide d'une chambre de diffusion [17]. Ces chambres de diffusion sont des petites capsules dans lesquelles on peut placer des cellules pour les isoler du milieu extérieur par une membrane de 0,22 $\mu \mathrm{m}$, ne laissant passer que les nutriments et les petites molécules. Lorsque les cellules C 6 traitées avec I'oligonucléotide antisens ont été injectées dans des rats $B D-I X$, aucune tumeur ne s'est développée (figure 3 ). Si les mêmes cellules sont injectées dans les souris nude, les tumeurs se développent mais avec un retard de croissance significatif par rapport aux celIules non traitées (Tableau I). Grâce à I'utilisation des chambres de diffusion, les auteurs ont montré que les cellules traitées in vitro par l'oligonucléotide antisens anti IGF-IR mourraient par apoptose très rapidement lorsqu'elles sont introduites dans des souris nude ou des rats BD-IX [17]. Cette apoptose apparaît plus importante in vivo qu'en culture in vitro. Le même résultat a été obtenu avec des clones cellulaires exprimant I'ARN antisens anti-IGF-IR. Le retard de $\mathrm{m} / \mathrm{s} \mathrm{n}^{\circ} 12$, vol. 17, décembre 2001 croissance des tumeurs in vivo est corrélé à la stimulation de cette apoptose. L'inhibition de I'IGF-IR par un ARN antisens ou un oligonucléotide antisens induit donc l'apoptose in vivo des tumeurs exprimant ce récepteur, confirmant ainsi le rôle anti-apoptotique de la voie de signalisation activée par l'IGF-IR.

Outre cette apoptose massive, I'inhibition de I'IGF-IR provoque également une réponse immuni- taire des rats traités impliquant des Iymphocytes CD 8+ (Tableau I). Selon les auteurs, des peptides partageant des caractéristiques communes avec les peptides portés par le complexe majeur d'histocompatibilité de classel ( $\mathrm{CMH} \mathrm{I),} \mathrm{pour-}$ raient, en s'échappant à travers les pores des chambres de biodiffusion, être cytotoxiques pour les tumeurs et également immunogéniques [17].

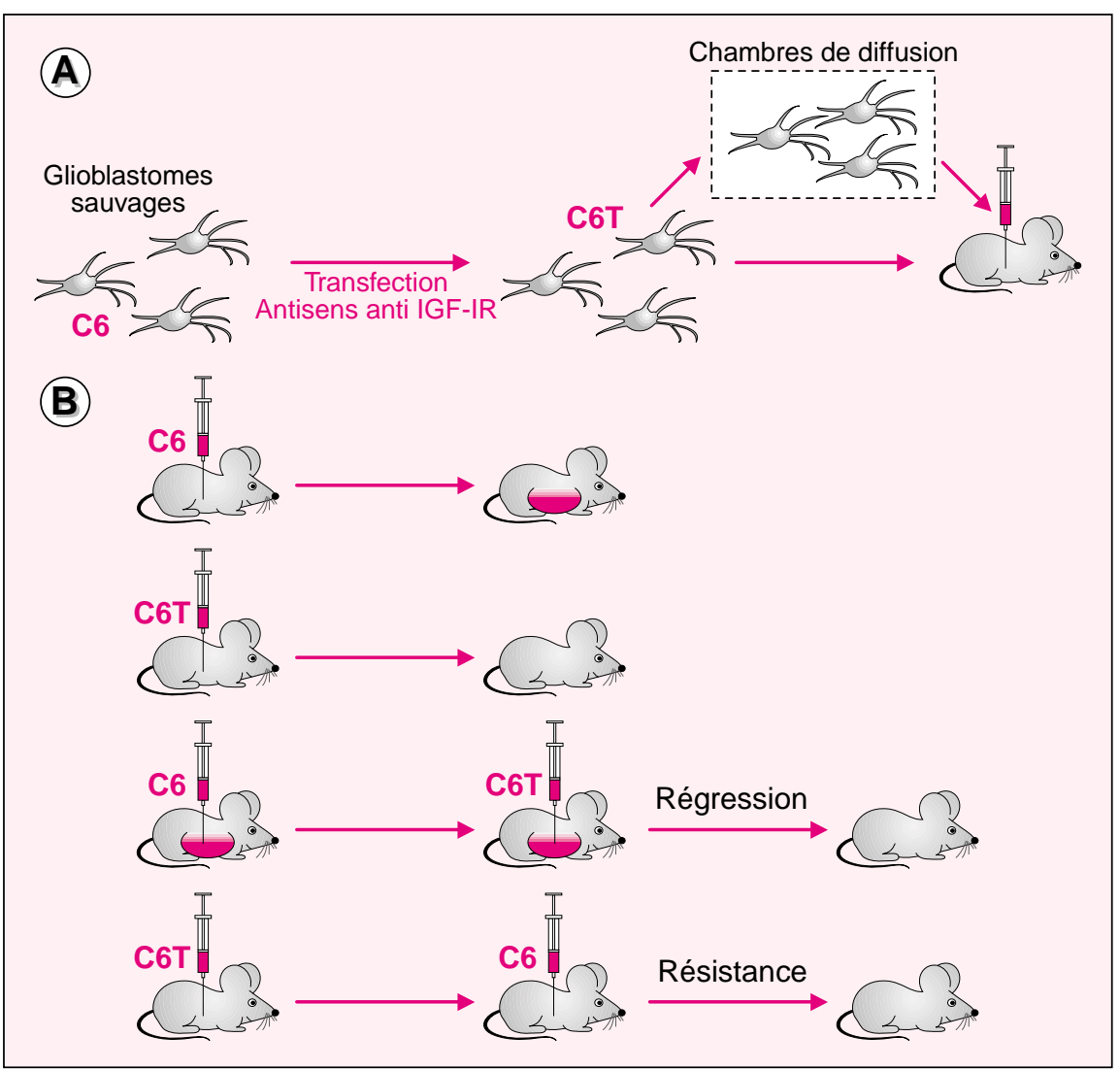

Figure 3. Blocage de la tumorigenèse des glioblastomes in vivo par l'utilisation d'antisens anti IGF-IR. A. Les cellules de glioblastomes C6 sont transfectées soit avec un plasmide codant pour l'ARN antisens anti IGF-IR, soit avec un oligonucléotide antisens anti IGF-IR. Les cellules transfectées C6T sont ensuite injectées directement chez les animaux, ou placées dans des chambres de biodiffusion. B. Les glioblastomes C6 induisent le développement de tumeurs chez le rat BD-IX et la souris nude. Les cellules C6T ne sont plus tumorigènes chez le rat. Chez le rat, elles sont toutes entrées en apoptose. Chez la souris nude, les tumeurs ont un retard de croissance corrélé à la fraction de cellules survivantes. Lorsque les cellules C6T (encapsulées ou non) sont injectées à des rats présentant une tumeur pré-établie, on observe une régression tumorale. Si les rats sont traités préalablement avec les C6T, une seconde injection de cellules C6 non transfectées ne conduit à aucune tumeur. Cette résistance à une seconde injection tumorale est dépendante de la présence de lymphocytes $\mathrm{CD}^{+}[17]$. 
Tableau I. Injections de cellules de glioblastomes à des rats BD-IX et des souris nude.

\begin{tabular}{|lccc|}
\hline & Cellules C6 & \multicolumn{2}{c|}{ Cellules C6T } \\
& Rat/Souris & Rat BD-IX & Souris nude \\
Tumeurs & & - & + \\
Expression de I'IGF-IR & ++ & - & - \\
Apoptose des cellules injectées & - & ++ & ++ \\
Réponse immune CD8+ & - & + & - \\
\hline
\end{tabular}

\section{Le ligand IG F-I et les tumeurs}

Une induction similaire de l'apoptose et de la réponse immunitaire anti-tumorale est observé lorsque le ligand du récepteur, I'IGF-I, est inhibé par un ARN antisens dans des glioblastomes C6 [9, 19]. L'inhibition de l'IGF-I coïncide avec I'apparition des antigènes du $\mathrm{CMH}$ I et de la molécule co-activatrice B7 au niveau de la membrane des cellules C6 exprimant I'ARN antisens anti-IGF-I [12]. Dans ce cas, ces protéines membranaires pourraient participer à l'induction de la réponse immunitaire anti-tumorale. Dans un autre modèle, le système syngénique d'hépatocarcinome de rat LFC2A, on observe aussi une inhibition de la croissance tumorale in vivo [12]. L'injection de la lignée d'hépatocarcinome de rat dont l'IGF-I est neutralisé provoque en effet moins de tumeurs que les cellules non transfectées et, dans $60 \%$ des cas, induit une régression des tumeurs préétablies. La réponse au traitement est cependant partielle comparée à celle obtenue avec les glioblastomes [12]. Une analyse plus détaillée a permis de montrer que les cellules d'hépatocarcinome exprimant I'ARN antisens anti IGF-I, surexprimaient aussi I'antigène du $\mathrm{CMH}$ de classe I, mais pas les molécules B7. L'absence de cette protéine membranaire pourrait expliquer la réponse partielle du traitement avec les ARN anti IGF-I. Très récemment, I'approche ARN antisens anti IGF-I a été couplée à l'expression de la molécule B7.1 afin d'obtenir un effet anti-tumoral [20]. Les cellules murines d'hépatomes et de carcinomes du côlon, du fait de leur développement de tumeurs chez des souris nude, scid et syngéniques [20]. L'expression de l'ARN antisens anti IGF-I et de la protéine B7.1 dans ces cellules (par double transfection) a permis de disposer d'un vaccin cellulaire provoquant l'éradication de tumeurs préexistantes de même origine dans des modèles de souris syngéniques [20]. Afin de démontrer le rôle fondamental joué par la réponse immunitaire dans l'éradication des tumeurs chez ces souris syngéniques, des expériences similaires ont été effectuées chez des souris immunodéprimées (scid et nude). De façon attendue, aucune régression de tumeur préexistante n'a été observée après injection de cellules exprimant B7.1 et I'antisens anti IGF-I.

\section{Essais pilotes chez l'homme}

Les astrocytomes malins sont des tumeurs intracrâniennes humaines qui résistent ou répondent partiellement aux traitements classiques de chirurgie, de radiation, et de chimiothérapie. Les malades atteints de glioblastomes décèdent rapidement, la médiane de survie étant de 48 semaines. L'expression de I'IGF-IR et de son ligand IGF-I apparaît légèrement supérieure dans des gliomes humains par rapport à des tissus normaux du cerveau (pour revue sur l'expression de la famille des IGF dans les tumeurs humaines, voir [4]). Après les résultats encourageants obtenus chez les rats et les souris nude avec les oligonucléotides antisens anti IGF-IR, des études pilotes chez l'homme ont été entreprises pour analyser la faisabilité d'un tel traitement anti-tumoral dans le cas de glioblastomes. Douze patients, atteints de glioblastomes ou d'astrocytomes anaplasiques ayant résisté aux traitements thérapeutiques classiques et exprimant IGF$I R$, ont participé à cet essai clinique [6]. Après exérèse partielle des tumeurs, les cellules tumorales sont dissociées puis placées en culture in vitro. Les cellules adhérentes obtenues sont traitées directement avec I'oligonucléotide antisens anti IGFIR pendant au moins 6 heures, puis collectées et placées dans des chambres de diffusion. Plusieurs chambres, contenant chacune un million de cellules, sont réimplantées de façon autologue dans une poche de l'abdomen du patient. Après 24 heures, les capsules de diffusion sont récupérées et leur contenu analysé. Dans tous les cas, les cellules tumorales récupérées sont incapables de proliférer à nouveau en culture, mais $2 \%$ de ces cellules sont toutefois intactes excluant le bleu trypan. L'introduction des chambres de diffusion contenant ces cellules tumorales traitées par l'oligonucléotide anti IGF-IR a conduit à des améliorations cliniques et radiographiques remarquables chez huit des douze patients traités (trois parmi ces huit ont été traités deux fois). Chez deux patients, les tumeurs primaires ont disparu temporairement. Malgré l'observation d'une rechute tumorale et une seconde chirurgie, la survie globale de ces deux patients semble avoir augmenté après ce traitement [6]. Comme le soulignent les auteurs, deux critiques majeures peuvent être portées sur ces premiers résultats chez I'homme. Tout d'abord, on ne peut exclure que la réponse antitumorale observée soit purement fortuite, sans rapport avec le traitement. De plus, s'il s'agit bien d'une réponse biologique au traitement, le mécanisme anti-tumoral impliqué reste à identifier [6]. Connaissant les résultats obtenus chez le rat, les auteurs ont d'abord recherché l'existence d'une réponse immunitaire induite par le traitement avec les chambres de diffusion. Si quelques patients ont bien présenté une infiltration lymphocytaire au niveau de la tumeur, celle-ci n'est pas observée chez tous les patients et il reste diffi- 
cile de conclure de façon sûre à l'existence d'un mécanisme immunodépendant. Les auteurs proposent également que les cellules des glioblastomes, traitées ex vivo avec l'oligonucléotide antisens et réinjectées, meurent par apoptose en libérant dans le milieu des facteurs peptidiques [6]. Dans cette hypothèse, ces petits peptides seraient d'une part cytotoxiques pour la tumeur et d'autre part capables de déclencher une réponse immunitaire anti-tumorale chez le patient.

\section{Conclusions}

L'inhibition de la croissance tumorale et l'induction de l'apoptose obtenues en inhibant l'expression de I'IGF-I ou de son récepteur dans des tumeurs animales, notamment dans des glioblastomes de rat, a conduit naturellement les chercheurs à tester ces approches antisens en thérapeutique humaine. Ainsi, des essais cliniques utilisant des ARN antisens anti IGF-I sont actuellement en cours chez des patients atteints de glioblastomes à Cleveland (USA), et de carcinomes du colon et du sein à Cracovie (Pologne). Les premiers essais cliniques utilisant des oligonucléotides antisens anti IGF-IR appliqués ex vivo sur les tumeurs chez des patients atteints de glioblastomes ont démontré la faisabilité et la non toxicité du traitement [6]. Si des améliorations transitoires radiographiques et cliniques ont même été observées chez certains patients [6], il convient toutefois de rester prudent vis-à-vis de ces réponses biologiques qui pourraient $n$ 'être que fortuites [6]. D'autres essais cliniques restent à effectuer avant de savoir si cette approche thérapeutique peut non seulement améliorer la vie du patient, mais aussi détruire complètement les tumeurs et empêcher toute rechute tumorale. Notons que ces stratégies ARN et oligonucléotides antisens pourraient également s'appliquer à d'autres cancers, comme les cancers de la prostate, du sein, et des poumons où il a été montré que le récepteur de I'IGF-I jouait un rôle essentiel $[2,4,13$, 21]

\section{RÉFÉRENCES}

1. Hanahan D, Weinberg RA. The hallmarks of cancer. Cell 2000; 100: 57-70.

2. Werner $H$, Le Roith D. New concepts in regulation and function of the insulin-like growth factors: implications for understanding normal growth and neoplasia. Cell Mol Life Sci $2000 ; 57$ : 932-42.

3. Adams TE, Epa VC, Garrett TP, Ward CW. Structure and function of the type 1 insulin-like growth factor receptor. Cell $\mathrm{M} \mathrm{ol}$ Life Sci 2000; 57 : 1050-93.

4. Khandwala HM, McCutcheon IE, Flybjerg $A$. Friend $K E$. The effects of insulin-like growth factors on tumorigenesis and neoplastic growth. Endocr Rev 2000; 21 : 215-44.

5. Resnik JL, Reichart DB, Huey K, Webster N), Seely BL. Elevated insulin-ike growth factor I receptor autophosphorylation and kinase activity in human breast cancer. Cancer Res 1998; 58 : 1159-64.

6. Andrews DW, Resnicoff M, Flanders AE et al. Results of a pilot study involving the use of an antisense oligodeoxynucleotide directed against the insulin-like growth factor type I receptor in malignant astrocytomas. J Clin Oncol 2001; 19: 2189-200.

7. Weiss B, Davidkova G, Zhou LW. Antisense RNA gene therapy for studying and modulating biological processes. Cell Mol Life Sci 1999; 55 : 334-58.

8. Myers KJ, Dean NM. Sensible use of antisense: how to use oligonucleotides as research tools. Trends Pharmacol Sci 2000 . $21: 19-23$

9. Trojan J, Johnson TR, Rudin SD, Ilan J, Tykocinski ML, Ilan J. Treatment and prevention of rat glioblastoma by immunogenic C 6 cells expressing antisense insulin-like growth factor I RNA. Science 1993; 259: 94-7.

10. Resnicoff M, Sell C, Rubini M, et al. Rat glioblastoma cells expressing an antisense RNA to the insulin-like growth factor-1 (IGF-1) receptor are nontumorigenic and induce regression of wild-type tumors. Cancer Res $1994 ; 54: 2218-22$.

11. Rininsland $F$, Johnson TR, Chernicky $C L$, Schulze E, Burfeind P, Ilan J. Suppression of insulin-like growth factor type I receptor by a triple-helix strategy inhibits IGF-- transcription and tumorigenic potential of rat C6 glioblastoma cells. Proc Natl A cad Sci USA 1997; 94 : 5854-9.

12. L afarge-Frayssinet C, Duc HT, Frayssinet $C$, et al. Antisense insulin-like growth factor I transferred into a rat hepatoma cell line inhibits tumorigenesis by modulating major histocompatibility complex I cell surface expression. Cancer Gene Ther 1997; 4: 27685

13. Lee CT, Wu S, Gabrilovich D, et al Antitumor effects of an adenovirus expressing antisense insulin-like growth factor I recep- tor on human lung cancer cell lines. Cancer Res 1996; 56: 3038-41.

14. Liu X, Turbyville T, Fritz A, Whitesell L. Inhibition of insulin-like growth factor $i$ receptor expression in neuroblastoma cells induces the regression of established tumors in mice. Cancer Res 1998; 58: 5432-8.

15. Wraight Cl, White PJ, McKean SC, et al. Reversal of epidermal hyperproliferation in psoriasis by insulin-like growth factor I receptor antisense oligonucleotides. $\mathrm{Nat}$ Biotechnol 2000; 18: 521-6.

16. Akhtar S, Hughes MD, Khan A, et al. The delivery of antisense therapeutics. Adv Drug Deliv Rev 2000; 44: 3-21.

17. Resnicoff M. Antitumor effects elicited by antisense-mediated down regulation of the insulin-like growth factor I receptor (review). Int J M ol M ed 1998; 1: 883-8.

18. Beutler A, Banck MS, Aguzzi A, Wedekind $D$, Hedrich $H$ J. Curing rat glioblastoma: immunotherapy or graft rejection? Science 1997; 276: 20-21.

19. U pegui-Gonzalez LC, Francois IC Ly A, Trojan ]. The approach of triple helix formation in control of gene expression and the treatment of tumors expressing IGF-I. Adv Exp M ed Biol 2000; 465: 319-32.

20. Liu $Y$, Wang $\mathrm{H}$, Zhao J, et al. Enhancement of immunogenicity of tumor cells by cotransfection with genes encoding antisense insulin-like growth factor-1 and B7.1 molecules. Cancer GeneTher 2000; 7: 456-65.

21. Chernicky CL, Yi L, Tan H, Gan SU, Ilan J. Treatment of human breast cancer cells with antisense RNA to the type I insulin-like growth factor receptor inhibits cell growth, suppresses tumorigenesis, alters the metastatic potential, and prolongs survival in vivo. Cancer $\mathrm{G}$ ene Ther 2000; 7: 384-95.

22. Li SL, Liang SI, Guo N, Wu AM, FujitaYamaguchi $Y$. Single-chain antibodies against human insulin-like growth factor I receptor: expression, purification, and effect on tumor growth. Cancer Immunol Immunother 2000; 49: 243-52.

23. Reiss $K, T u X$, Romano $G$, Peruzzi $F$, Wang $J Y$, Baserga $R$. Intracellular association of a mutant insulin-like growth factor receptor with endogenous receptors. Clin Cancer Res 2001; 7: 2134-44.

24. Prager D, Li HL, Asa S, Melmed S. Dominant negative inhibition of tumorigenesis in vivo by human insulin-like growth factor I receptor mutant. Proc Natl A cad Sci USA 1994: 91 : 2181-5.

25. Pietrzkowski Z, Mulholland G, Gomella $L$, Jameson BA, Wernicke $D$ Baserga $R$. Inhibition of growth of prostatic cancer cell lines by peptide analogues of insulin-like growth factor 1 . Cancer Res 1993; 53: 1102-6. 


\section{RÉFÉRENCES}

26. Navab R, Chevet E, Authier F, Di Guglielmo GM, Bergeron Jj, Brodt P. Inhibition of endosomal insulin-like growth factor-I processing by cysteine proteinase inhibitors blocks receptor-mediated functions. Biol Chem 2001; 276: 13644-9.

27. Delafontaine $P, M e n g X P, K u L, D u J$. Regulation of vascular smooth muscle cell insulin-like growth factor I receptors by phosphorothioate oligonucleotides. Effects on cell growth and evidence that sense targeting at the ATG site increases receptor expression. J Biol Chem 1995; 270 : 14383-8.
28. Ly A, Duc HT, Kalamarides M et al. Human glioma cell's transformed by IGF-I triple helix technology show immune and apoptotic characteristics determining cell selection for gene therapy of glioblastoma. Mol Pathol 2001: 54 : 230-9.

\section{TIRÉS À PART}

J.C. François.

\section{Jean-Christophe François}

Laboratoire de biophysique, Inserm U. 201, Cnrs UM R 8646, M uséum national d'histoire naturelle, 43, rue Cuvier, 75231 Paris Cedex 05 , France.

E-mail : francois@mnhn.fr

\section{Jerzy Trojan}

Laboratoire de neurologie, Inserm EPI9935/ Université Paris VII, H ôpital Robert-D ebré, 48 , boulevard Serurier, 75019 Paris, France.

\section{BRÈVES}

La marque du NO : il n'y a que nitrite qui vaille. Le monoxyde d'azote (NO) est un vasodilatateur très puissant synthétisé par l'endothélium vasculaire à partir d'arginine, réaction catalysée par la NO-synthase endothéliale (eNOS). La détection du NO pose aux expérimentateurs un problème majeur en raison de la brièveté de sa demi-vie, de l'ordre de quelques secondes. En recherche clinique, analyser des variations de synthèse de NO, c' est-à-dire des variations de l'activité de la eNOS, revient donc à mesurer les concentrations de métabolites stables, nitrite $\left(\mathrm{NO}_{2}{ }^{-}\right)$et surtout nitrate $\left(\mathrm{NO}_{3}{ }^{-}\right)$, terme de la chaîne d'oxydation du NO. Parce que la concentration plasmatique de nitrate est 100 fois supérieure à celle de nitrite, c'est habituellement elle (ou la somme nitrate + nitrite) qui est mesurée... probablement à tort. Le groupe de Malte Kelm [1] apporte des arguments qui plaident en faveur d'une détection locale de nitrite lorsqu'une modification du débit sanguin d'origine endothéliale est recherchée. En utilisant un protocole de modification du tonus vasculaire de l'avant-bras, protocole classique en pharmacologie clinique cardio-vasculaire, les auteurs montrent que l'augmentation du débit sanguin local, induite par la perfusion d'acétylcholine qui stimule la eNOS, entraîne une augmentation parallèle de nitrite dans l'effluent veineux alors que la concentration de nitrate ne change pas. L'inhibition de la eNOS par le LNMMA provoque, au contraire, une chute du débit sanguin et une diminution contemporaine de la concentration de nitrite. Là encore, la concentration de nitrate est inchangée. Les concentrations systémiques de nitrate et de nitrite sont restées stables au cours de l'ensemble de ces conditions expérimentales. Enfin, contrairement à ce que des travaux antérieurs avaient laissé entrevoir, aucun effet vasodilatateur propre du nitrite n'est mis en évidence. Cette étude fournit un outil précis pour la recherche et l'exploration de situations pathologiques caractérisées par une dysfonction endothéliale.

[1. Lauer T, et al. Proc Natl Acad Sci USA $2001 ; 98$ : 12814-9.]

므 bienfaits de l'inactivité de CCR5. CCR5 est un récepteur pour les chimiokines RANTES, MIP-1 $\alpha$ et MIP-1 $\beta$. Or environ $1 \%$ de la population caucasienne est homozygote pour une forme du gène CCR 5 délétée de 32 paires de bases (CCR5 $\Delta 32$ ). CCR5 32 code pour un récepteur inactif, et les porteurs de cette forme mutée sont protégés de l'infection par le virus HIV, dont CCR5 est un des co-récepteurs. Les hétérozygotes se comportent comme les porteurs du gène non délété. Or il s'avère que CCR5 532 a d'autres conséquences médicales bénéfiques: une étude du Lancet [1] révèle que parmi les patients ayant subi une transplantation rénale, ceux qui sont homozygotes pour la forme CCR5 $\triangle 2$ ont une survie statistiquement plus longue que les patients dont CCR5 est soit muté sur un seul allèle, soit non muté. L'étude a porté sur 1227 patients allemands transplantés, dont $21(1,7 \%)$ étaient homozy- gotes pour le gène CCR $5 \Delta 32$, et $20 \%$ hétérozygotes. Le suivi à long-terme de 576 de ces patients, dont les 21 patients CCR5 $\triangle 32$, montre que la perte fonctionnelle du rein greffé n'est survenue que chez un seul des patients CCR $5 \Delta 32$ (même si des signes histologiques de rejet ont été identifiés chez 9), contre 78 dans le groupe contrôle, une différence statistiquement significative. Cette différence peut s'expliquer: lors d'un épisode de rejet, le rein greffé sécrète localement les chimiokines RANTES et MIP- $1 \alpha$, ligands de CCR5, qui attirent lymphocytes et macrophages du receveur, entretenant une réaction inflammatoire et immunitaire delètère. L'inactivité de CCR5 chez les patients $C C R 5 \Delta 32$ les protègerait de cette réaction inflammatoire. De fait, la neutralisation de CCR5 ou de CCR 1 (qui lie aussi RANTES et MIP-1 $\alpha$ ) diminue les rejets d'organe (cœur) dans des modèles animaux expérimentaux. On peut donc faire de l'existence de CCR $5 \Delta 32$ un critère pronostique après transplantation rénale, mais il pourrait aussi être utilisé pour identifier les patients qui pourraient bénéficier d'un traitement immunosuppresseur plus léger, ou ceux pour lesquels la sélection de compatibilité HLA du greffon serait moins draconienne. II n'est pas exclu que cette délétion protège également des conséquences d'autres maladies auto-immunes ou allergiques, comme l'asthme, où les chimiokines ont un rôle prédominant.

[1. Fischereder $M$, et al. Lancet 2001; 357 : 1758-61.] 